\title{
The effect of periodic segmentation cracks on the interfacial debonding: Study on interfacial stresses
}

\author{
Chen-Wu Wu ${ }^{\mathrm{a}, \mathrm{b}, *}$, Guang-Nan Chen ${ }^{\mathrm{a}}$, Kun Zhang ${ }^{\mathrm{a}}$, Geng-Xing Luo ${ }^{\text {a }}$, Nai-Gang Liang ${ }^{\mathrm{a}}$ \\ a Institute of Mechanics, Chinese Academy of Sciences, Beijing 100080, PR China \\ ${ }^{\mathrm{b}}$ Graduate School of the Chinese Academy of Sciences, Beijing 100049, PR China
}

Received 7 June 2005; accepted in revised form 17 November 2005

Available online 20 December 2005

\begin{abstract}
Hard coatings on relatively soft substrate always face the danger of debonding along the interface. Interfacial stresses are considered to be the initial driving force for the interfacial debonding of the relatively strong bonded coatings. Interfacial stresses due to the mismatch of strain between the coating and substrate are simulated with FEM firstly. The distribution of the interfacial stresses is achieved, which confirms an excessive stresses concentration near the interface end. Subsequently, the redistribution of interfacial stresses is calculated for a coating with periodic segmentation cracks. Results indicate that the distribution of interfacial stresses is altered greatly with the periodic segmentation cracks. To reveal the effect of the spacing of the periodic segmentation cracks on the distribution of interfacial stresses, different crack density is modeled within the coating. It is found that that the peak values of the interfacial stresses decrease with the increase of crack density, i.e. with reduction of spacing of segmentation cracks.
\end{abstract}

(C) 2005 Elsevier B.V. All rights reserved.

Keywords: Coatings; Periodic segmentation crack; Interfacial stresses; FEM; Crack density

\section{Introduction}

Hard coatings are often applied to softer, ductile substrates in order to provide abrasion, corrosion or thermal shock resistance. Such coatings are usually brittle and may fail by cracking in coating and debonding between the coating and substrate. Cracking and debonding occurs when there is excessive strain mismatch between the coating and substrate due to imposed thermomechanical loads or residual stresses [1-3]. The combination of the two materials with mismatch of properties across the interface always results in concentration of interfacial stresses [4], which will lead to initiation of debonding (as shown in Fig. 1). Fig. 1 shows that the interfacial cracking develops after the surface crack penetrate

\footnotetext{
* Corresponding author. Lab for Surface Modification, Institute of Mechanics, Chinese Academy of Sciences, Beijing 100080, PR China. Tel.: +86 10 62547527 1; fax: +86 1062545533 .

E-mail address: wcw_on@yahoo.com.cn (C.-W. Wu).
}

into the steel substrate. Note that the coating is electroplating chromium. The originations of such interfacial cracking are believed to be the result of the concentration of interfacial stresses. The concept to reduce the mismatch and then to decrease the interfacial stresses with all kinds of structure designs, such as multilayer coating etc., attracts the researchers for a long time.

Recently, Chen et al. [5] reported that pre-treating the substrate metal with discrete laser quenching before electroplating chromium, periodic segmentation cracks form in the coating, as shown in Figs. 2 and 3. Here, segmentation cracks are named for the vertical cracks penetrating at least half of the coating thickness. Fig. 2 shows that segmentation cracks form within the coating corresponding to the period of the laser quenching, i.e. one segmentation crack develop within every space between two adjacent pre-quenched regions. To reveal it in detail, the first left segmentation crack in Fig. 2 is magnified in Fig. 3. Surprisingly, the coatings with periodic segmentation cracks survived longer than intact ones, i.e. the former bearing more load cycles 


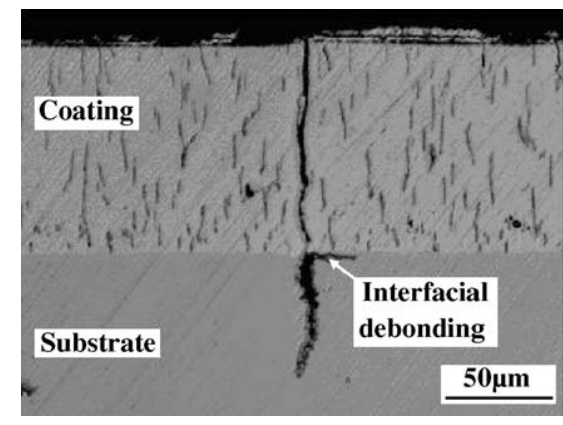

Fig. 1. Interficial debonding from cut through.

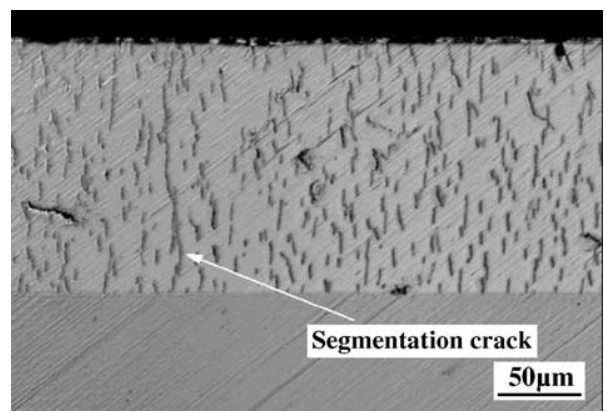

Fig. 3. Magnified profile of one segmentation crack. before debonding [5]. It is considered to be due to the beneficial effect of the periodical segmentation cracks on the interfacial debonding.

Actually, the positive effect of segmentation cracks has been reported for TBC by [6-8]. The columnar microstructure of some TBC provides a reduced stiffness in tension. Taylor [9] found that the initially cracked coatings have better thermal shock resistance than the initially perfect coatings. The effect of multiple cracking has also been studied for applications related to laminate composites by Schulz and Erdogan [10]. In their analytical study of periodic cracking of elastic coatings, they found that the surface stresses increase with the increase of crack spacing or substrate stiffness, and the decrease of crack length. The effect of the precracks in TBC on the interfacial cracks were simulated by Kokini et al. [11] and Zhou et al. [12], in which the effects of the density of the surface cracks on the energy release rate of the interfacial cracks were mainly considered. In all of these studies on the effect of the periodic cracking, the interfacial cracking is taken as being in existence. This is consistent with the fact of those coatings being relatively weakly bonded, in which the interfacial damage arises even during fabrication. However, as for the relatively strong bonded coating, there is a long timespan before the interfacial cracking initiates. Thus, the effect of segmentation cracks within the perfectly bonded coating on the interfacial stresses should be paid sufficient attention, which are not found to be reported up to now.

In this study, the interfacial stresses due to the mismatch of strain between the coating and substrate are simulated with FEM firstly. Subsequently, the redistribution of interfacial stresses is analyzed for a periodically cracked coating. To reveal the effect of the spacing of the periodic segmentation cracks on the distribution of interfacial stresses, different crack densities are modeled within the coating. Finally, some discussions are pro- vided to interpret the mechanism responsible for the above phenomena.

\section{The interfacial stresses between coating and substrate}

\subsection{Analysis and numerical model}

Due to the mismatch between the coating and substrate, the interfacial stresses will arise even if there are only loads in the plane paralleling to the interface. Aimed at the structure of the coating and substrate, a simple sketch is established as shown in Fig. 4. It shows schematically the interfacial stresses near the free end due to in-plane load. This is simplified as a plane strain problem, in which only the strains within plane OXY are considered.

To simulate the state of the coating during cooling, a static uniform tension was exerted on the substrate. One can understand easily that there are always tensile normal stresses in the interface near the free end when the substrate is being elongated, which is required with the moment equilibrium.

The geometry model is shown in Fig. 5 , where $L, t_{\mathrm{s}}$ and $t_{\mathrm{c}}$ is the width of specimen, thicknesses of the substrate and coating, respectively. And the substrate was uniformly extended $0.1 \mathrm{~mm}$ at either end. The finite element model was created in ANSYS, in which about 60,000 elements are used. When the edge effect is considered, the mesh was refined near the free end. The materials parameters used in this study are shown in Table 1. As the loads are self-equilibrated, no other constraints are required. Based on the assumption of perfect bonded along interface, the deformations crossing the interface meet the requirement of continuum in displacements. At the same time, the interfacial normal stress and interfacial shear stress should be identical for either coating or substrate, which is required by the continuity of the interfacial stresses for ideal perfect interface.

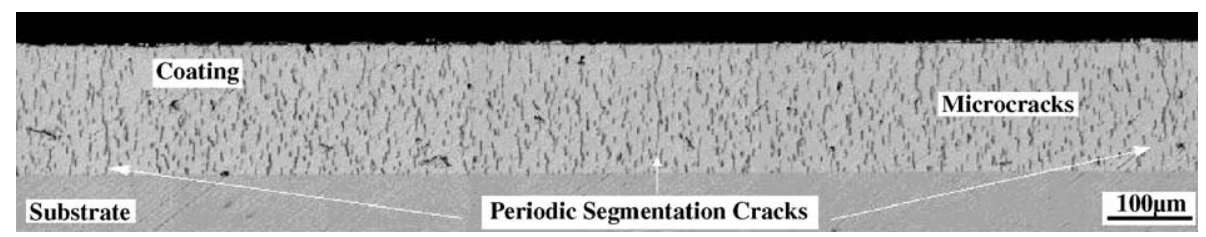

Fig. 2. The profile of the periodic segmentation cracks in coating (cross section). 


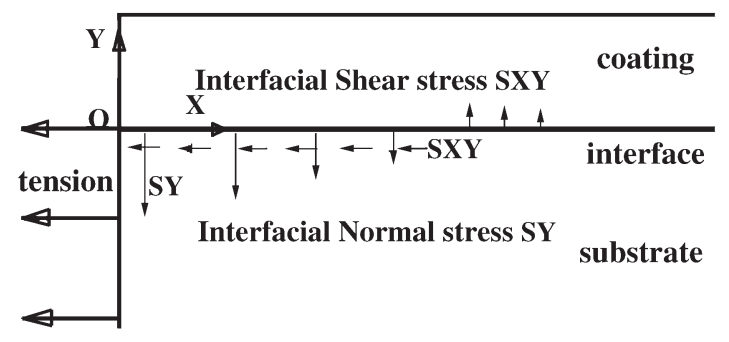

Fig. 4. The sketch of the interfacial stress near the free end.

\subsection{Results and discussion}

Fig. 6 shows the interfacial stresses near the free end, including interfacial normal stress and shear stress. Noting that $\bar{\sigma}$ is defined in the following expressions.

$$
\begin{aligned}
& \bar{\sigma}=\mathbf{E}^{*} \times \bar{\varepsilon} \\
& \mathbf{E}^{*}=\frac{\mathbf{E}_{\mathrm{c}} \mathbf{E}_{\mathrm{s}}}{\mathbf{E}_{\mathrm{c}}+\mathbf{E}_{\mathrm{s}}}, \\
& \bar{\varepsilon}=\varepsilon_{\mathrm{s}}^{0}-\varepsilon_{\mathrm{c}}^{0}
\end{aligned}
$$

Where, $\mathbf{E}_{\mathrm{c}}$ and $\mathbf{E}_{\mathrm{s}}$ are the moduli of the coating and substrate metal, respectively. And $\varepsilon_{\mathrm{s}}^{0}, \varepsilon_{\mathrm{c}}^{0}$ refer to the average strain of the substrate and coating in a free state, and the difference of which is named with $\bar{\varepsilon}$. In this study, the average strain of the substrate is $3.33 \%$, and that for the coating is 0 . Thus, $\bar{\varepsilon}$ is $3.33 \%$.

One can see that interfacial normal stress is prominent tension near the free end. And interfacial normal stress experiences compression farther away from the free end due to the state of static equilibrium. The shift law of the interfacial stresses is almost consistent with that of the analytic result by Zhang [14], where the interfacial stresses between bonded beams are solved with series methods. In Zhang's solutions it is confirmed that the interfacial normal stress have a maximum value at the free end and decreases sharply till altering its sign. The interfacial normal stress, especially when it is tension, is generally believed to be the initial driving force for the interfacial debonding. Thus it is beneficial for the durability of the coating to decrease interfacial stresses by some means or other.
Table 1

The material parameters of the two materials [13]

\begin{tabular}{llll}
\hline Item & Elastic modulus & Poisson's ratio & Yield stress \\
\hline coating & $300 \mathrm{e} 9 \mathrm{~Pa}$ & 0.15 & - \\
substrate & $200 \mathrm{e} 9 \mathrm{~Pa}$ & 0.3 & $800 \mathrm{e} 6 \mathrm{~Pa}$ \\
\hline
\end{tabular}

\section{The effect of periodic segmentation cracks on interfacial stresses}

\subsection{The morphology of the periodic segmentation cracks}

In order to investigate the effect of the periodic segmentation cracks on the interfacial debonding, the structures with different crack density, i.e. the spacing of cracks, are modeled. Preliminarily, the segmentation cracks running through whole thickness of the coating are analyzed. The series of crack density is $0,1,3,5,7,9,11,23$ cracks uniformly aligned within the coating, which segment the coating as 1, 2, 4, 6, 8, 10, 12, 24 fragments, respectively. The model is shown in Fig. 7, which indicates that the width of the structure is $6 \mathrm{~mm}$, and the displacement loading is $0.1 \mathrm{~mm}$ at either end of structure. It is simplified as a plane strain problem, i.e. only the strains within plane OXY are considered.

Similar to above analysis, the mesh near the free ends and crack tips is sufficiently refined to meet the accuracy requirement. The loading for every case is the same as mentioned above to study the interfacial stresses for the coatings of different densities of periodic segmentation cracks. In Fig. 7, the focused zone enclosed with dashed ellipse notes where the maximums of the interfacial stresses probably emerge.

\subsection{Results and discussion}

The results indicate that there are multiple extremes of interfacial stresses along the interface when there are periodic segmentation cracks in the coating, and the extreme always corresponding to the zones around segmentation crack tips, as shown in Fig. 8. This is for that every segmentation crack is accompanied with two free ends, which introduce concentration of interfacial stresses. The results shown in Fig. 8 correspond to the case when 3 periodic segmentation cracks in the coating are considered. Note that the abscissa for the

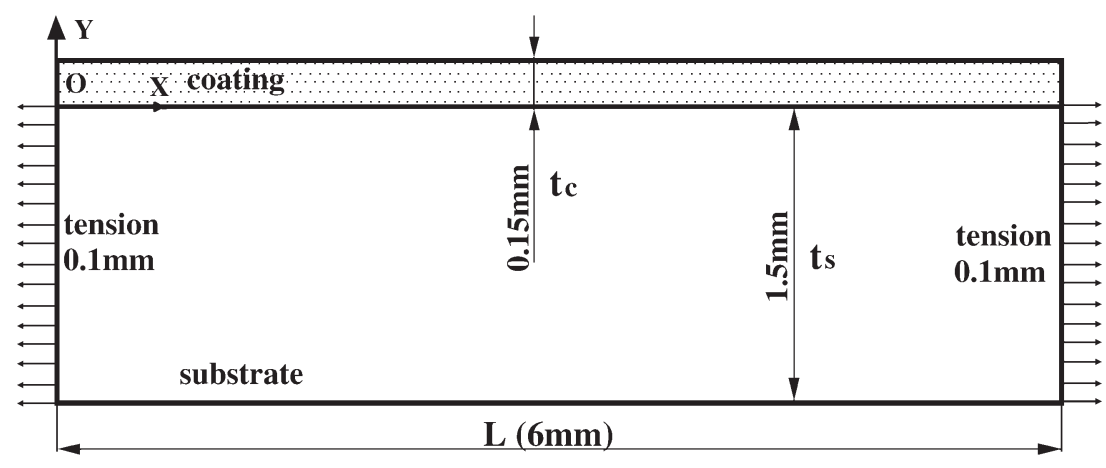

Fig. 5. The geometry model and dimensions for analysis. 


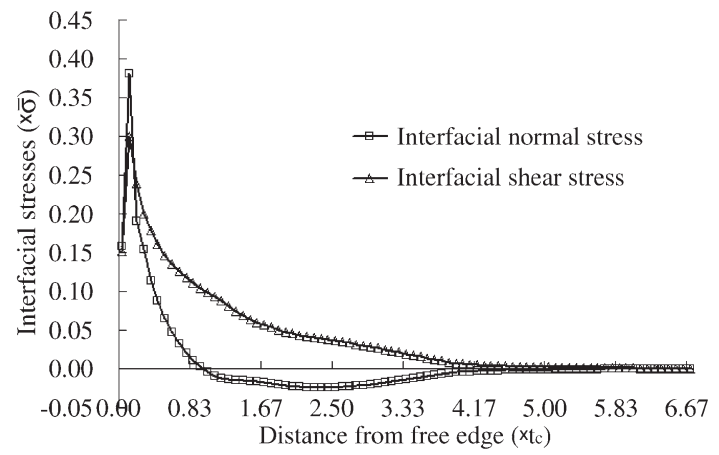

Fig. 6. Interfacial stresses along the distance from the free end.

curve presents half width of the structure only. It can be found that the maxima of the interfacial stresses are greatly less than that of the intact coating.

Fig. 9 shows the maximum interfacial stresses versus the density of the segmentation cracks. The spacing $(\boldsymbol{W})$ between every two adjacent segmentation cracks is determinate if the number $(\boldsymbol{D})$ of the cracks is fixed. And the relationship between $\boldsymbol{W}$ and $\boldsymbol{D}$ is given by the equation

$\boldsymbol{W} \times(\boldsymbol{D}+1)=\boldsymbol{L}$

Where $\boldsymbol{L}$ denotes the total width of the model, which is $6 \mathrm{~mm}$ for this analysis. The maximum interfacial normal stresses decrease sharply with the decreasing of spacing, particularly before the spacing falls to $1.2 \mathrm{~mm}$. It decreases about $53 \%$ in interfacial normal stress as the number of cracks increases from 0 to 5 , i.e. spacing from 6 to $1.2 \mathrm{~mm}$, and about $30 \%$ decrease in interfacial shear stress. The maximum interfacial stresses decrease more wildly after the density of cracks is greater than 9 per $6 \mathrm{~mm}$. This means that once the density of cracks reaches a threshold for special structure, the decrease rate of the maximum interfacial stresses will slow down.

The beneficial effect of the periodic segmentation cracks on the interfacial stresses, according to the present study, may be partly due to the decrease of the macroscopic stiffness of the coating so that it can endure more strain mismatch. The existence of the threshold also indicates that the positive effect is finite for a given structure and loading level.

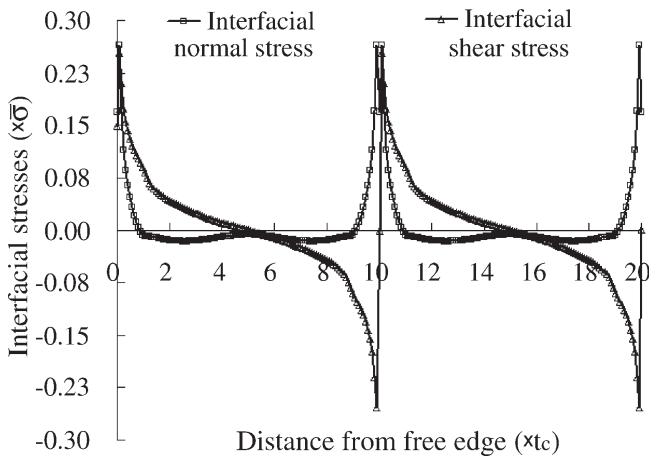

Fig. 8. Interfacial stresses along interface (for the case with 3 segmentation cracks within the whole width of the structure).

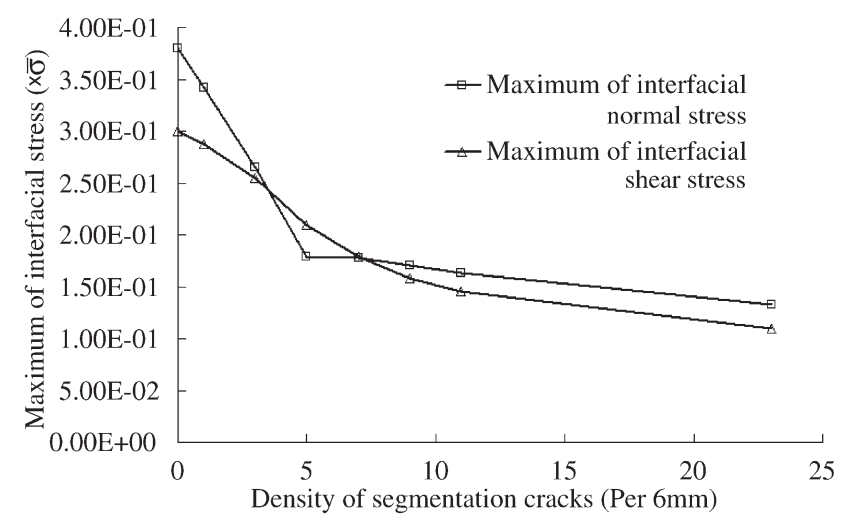

Fig. 9. Max interfacial stresses versus the density of segmentation cracks.

\section{Conclusions}

1. For coating bearing thermal shock, there are always interfacial normal tensile stresses at the interface during cooling. The interfacial stresses are prominent near the free end, which may be the initial driving force for interfacial debonding.

2. Periodic segmentation cracking in the coating can decrease the macroscopic stiffness of the structure, so that the coating can endure more strain mismatch. The thermal shock resistance of the coating may be improved with pre-producing periodic segmentation cracks in the coating.

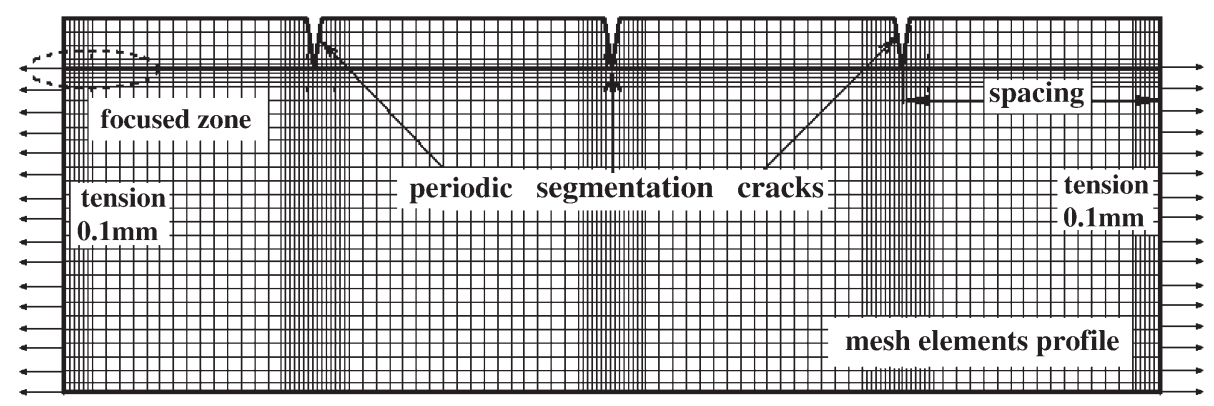

Fig. 7. The morphology of the segmentation cracks and the sketch of the mesh. 
3. The maximum interfacial stresses decrease sharply with the increase of crack density at the beginning. However, once the density of segmentation cracks reaches a threshold for a given structure and loading level, the shift rate of the maximum interfacial stresses will slow down.

\section{Acknowledgments}

The financial support provided by the National Natural Science Foundation of China (Grant NO. 50471087 and 50531060 ) is greatly acknowledged. Thanks are also due to all the other researchers of the Lab for Surface Modification, Institute of Mechanics, Chinese Academy of Science.

\section{References}

[1] K.A. Khor, Z.L. Dong, Y.W. Gu, Materials Letters 38 (1999) 437.

[2] S. Sopok, C. Rickard, S. Dunn, Wear 258 (2005) 659.

[3] J.W. Hutchinson, Z. Suo, Advances in Applied Mechanics 29 (1992) 63.
[4] Yujun Wen, Cemal Basaran, Mechanics of Materials 36 (2004) 369.

[5] Guang-Nan Chen, Geng-Xing Luo, Kun Zhang, Xiang-Yang Xu, Huan Shen, Hong-Min Yan, De-Rong Rao, Acta Armamentarii (China) 24 (2003) 6 .

[6] J.T. DeMasi-Marci, K. Gupta Dinesh, Surface and Coatings Technology 68 (1994) 1.

[7] A.H. Bartlett, R.D. Maschio, Journal of the American Ceramic Society 78 (4) (1995) 1018.

[8] T.A. Taylor, D.L. Appleby, A.E. Weatherill, J. Griffiths, Surface and Coatings Technology 43-44 (1-3) (1990) 470.

[9] T.A. Taylor, Surface and Coatings Technology 54-55 (1-3) (1992) 53.

[10] G.W. Schulz, F. Erdogan, International Journal of Solids and Structures 35 (28-29) (1998) 3615.

[11] Klod Kokini, Anuradha Banerjee, Thomas A. Taylor, Materials Science \& Engineering. A, Structural Materials: Properties, Microstructure and Processing 323 (1-2) (2002) 70 .

[12] Bin Zhou, Klod Kokini, Surface and Coatings Technology 187 (2004) 17.

[13] Z.X. Zhang, Handbook of Enginery Metal Materials, Weapon Industry Publisher, Beijing (China), 1989, p. 446.

[14] Fu Fan Zhang, The Interfacial Stresses in Composites, Higher Education Press, Beijing (China), 1993, p. 28 